\title{
BIOCHAR POTENTIAL IN RECLAIMING DEGRADED SOILS
}

\author{
Paloma Campos ${ }^{1}$, Ana Z. Miller², Heike Knicker ${ }^{1}$, José María De la Rosa ${ }^{1}$
}

1. Instituto de Recursos Naturales y Agrobiología de Sevilla (IRNAS-CSIC), Av. Reina Mercedes 10, 41012, Sevilla, Spain 2. HERCULES Laboratory, University of Évora, Évora, Portugal

pcampos@irnas.csic.es 
Soil pollution: excess of an element or compound

environmental risks

toxic response on biota and humans

The Aznalcóllar Mine accident

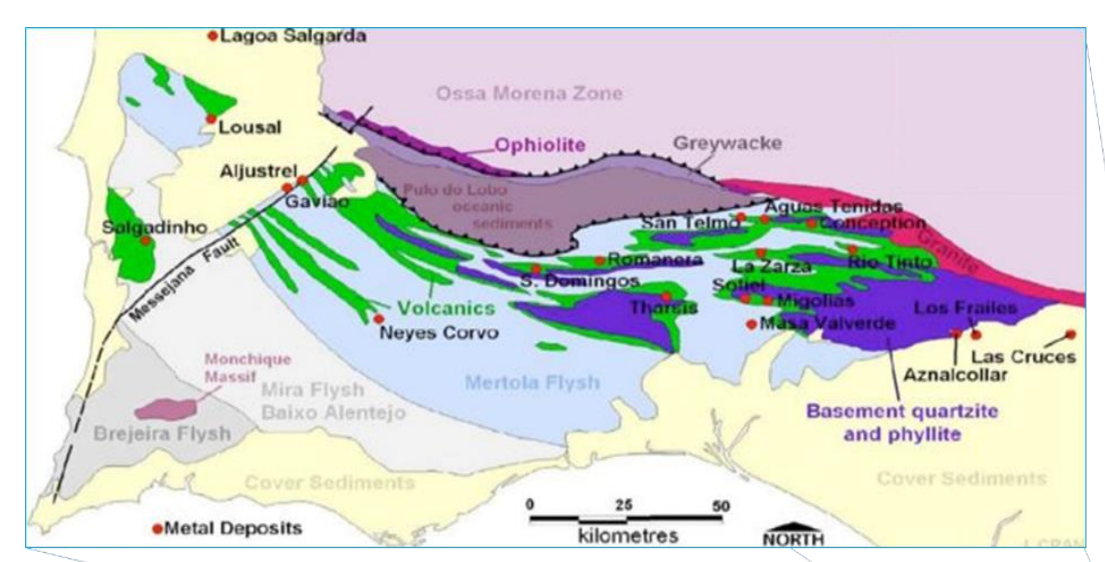

$25^{\text {th }}$ April 1998 (3:30 am)

- 4 millions $\mathrm{m}^{3}$ acidic water

- 2 millions $\mathrm{m}^{3}$ toxic mud
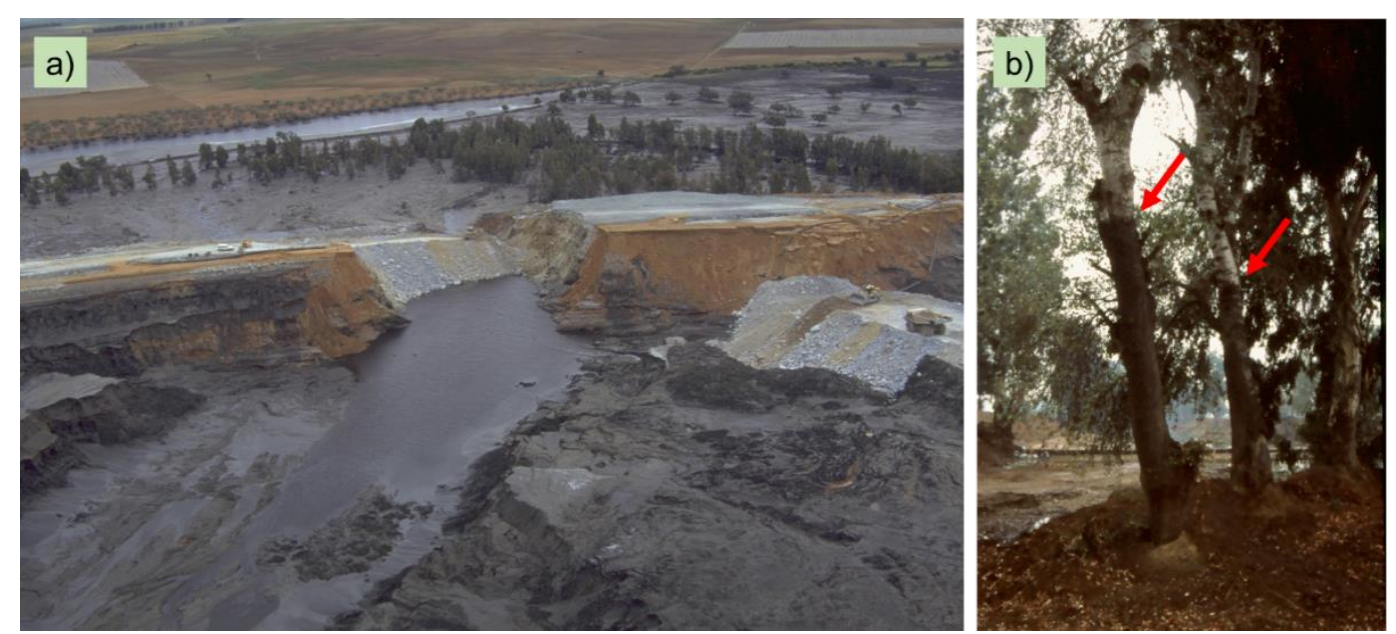

a) Aerial view of the mine-tailing spill of Aznalcóllar (With permission from Greenpeace(C); b) The flood reached at some points up to 3 meters high (Author: P. Arambarri).
Geological map of the Iberian Pyrite Belt and metal deposits (Adapted from Espi et al., 2008).

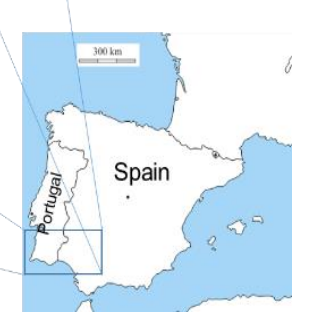




\section{The Aznalcóllar Mine Accident}

pcampos@irnas.csic.es

Total surface affected: 4286 ha

$400 \mathrm{~m}$ at both sides of the river

$\Longrightarrow 2$

Mud: along $40 \mathrm{~km}$ down river

$\longrightarrow \rightleftharpoons$

Water: along $60 \mathrm{~km}$ down river

$\longrightarrow$

Height of the muds from $\mathrm{cms}$ to $1.5 \mathrm{~m}$

30 tons of dead fishes

$\longrightarrow$
Sludge and dead

fauna removal
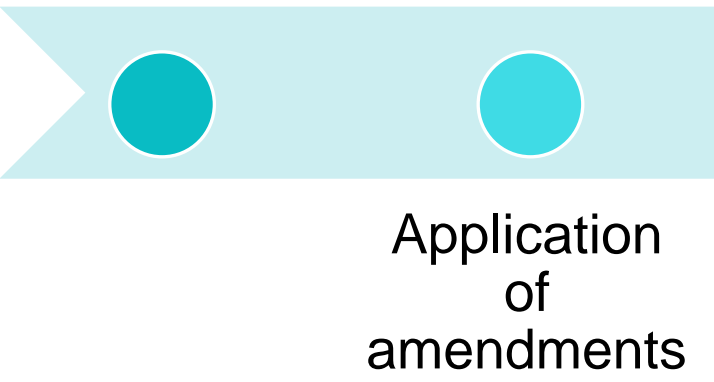

\section{We propose:}

BIOCHAR AS AMENDMENT FOR TRACE ELEMENT CONTAMINATED SOIL

For its properties:

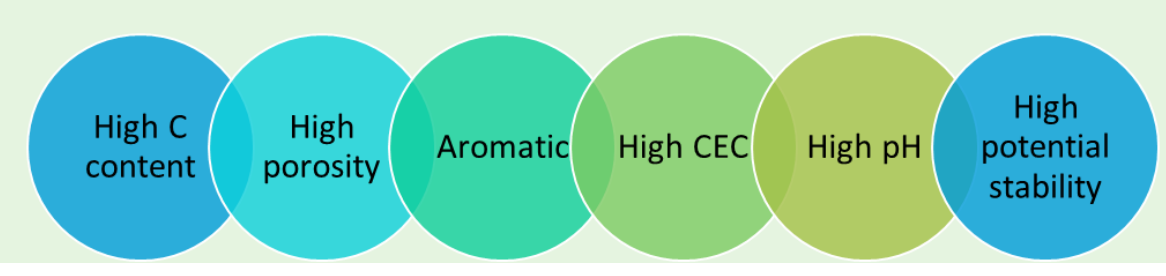

200 ha of soils still contaminated with trace elements
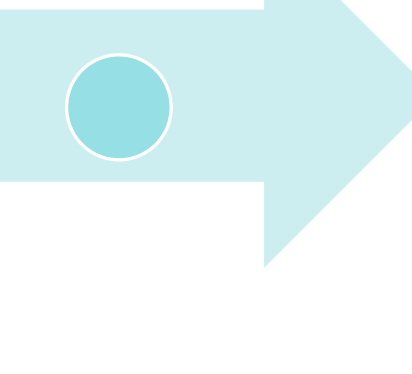
Biochar (BC) is the carbonaceous solid residue produced through the thermochemical conversion (pyrolysis) of biomass under low $\mathrm{O}_{2}$ atmosphere.
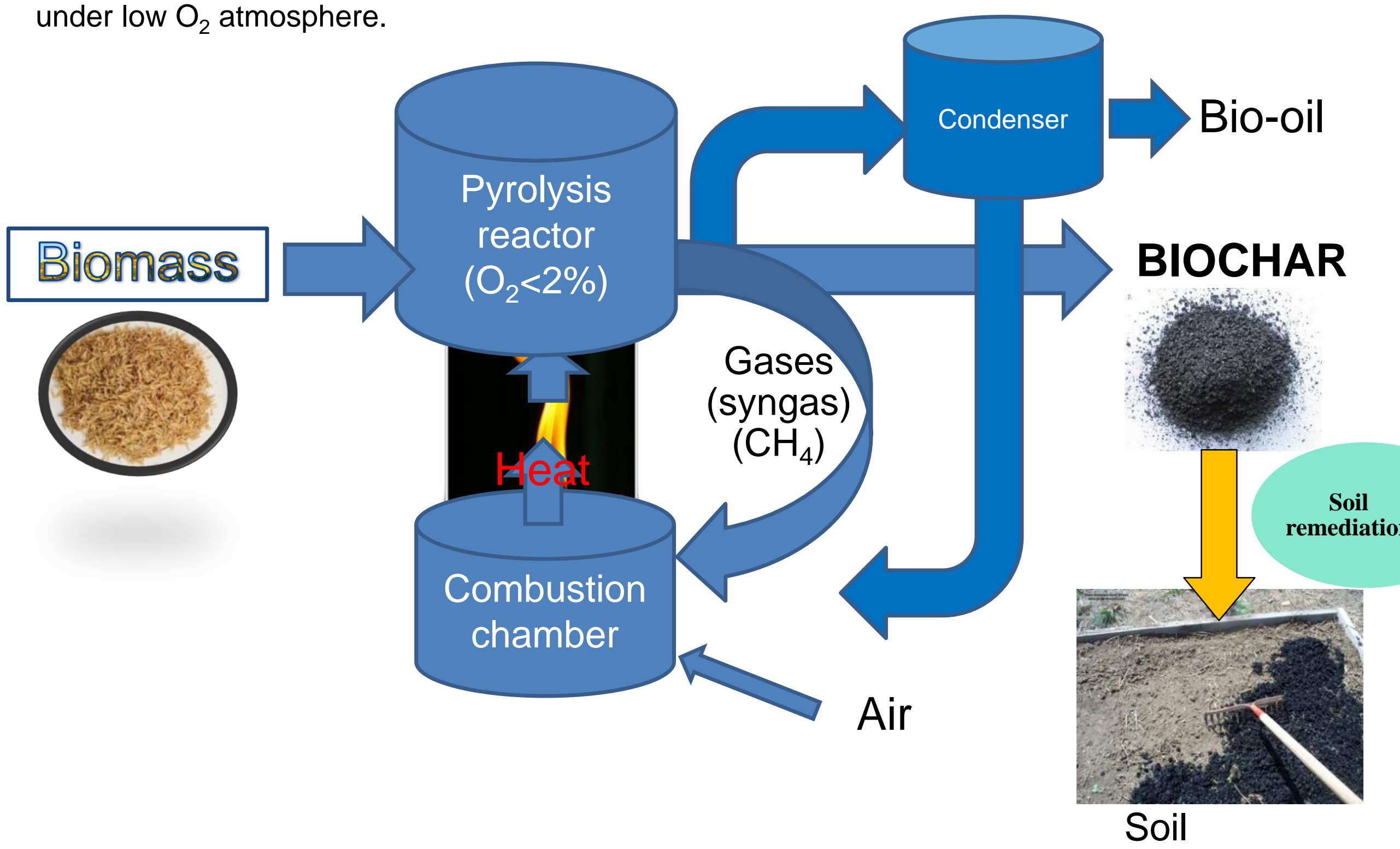

Management of organic wastes 


\section{Field experiment. In situ remediation}

pcampos@irnas.csic.es

Green Corridor of Guadiamar (25 km north Seville, SW Spain)

2 soils (clay loam soil - Fluvisols):

MPS: Moderately Polluted Soil

HPS: High Polluted Soil

\begin{tabular}{|cccccc|}
\hline & $\begin{array}{c}\text { WHC } \\
(\%)\end{array}$ & $\begin{array}{c}\text { Bulk } \\
\text { density } \\
(\mathbf{g} / \mathbf{m l})\end{array}$ & $\begin{array}{c}\mathbf{p H} \\
\left(\mathbf{C a C l}_{2}\right)\end{array}$ & $\begin{array}{c}\text { EC } \\
(\boldsymbol{\mu} \mathbf{S} / \mathbf{c m})\end{array}$ & $\% \mathbf{C}$ \\
\hline HPS & 32.73 & 1.1 & 3.62 & 3610 & 0.24 \\
\hline MPS & 51.45 & 1.2 & 6.47 & 1809 & 0.29 \\
\hline
\end{tabular}

MPS - Moderately Polluted Soil

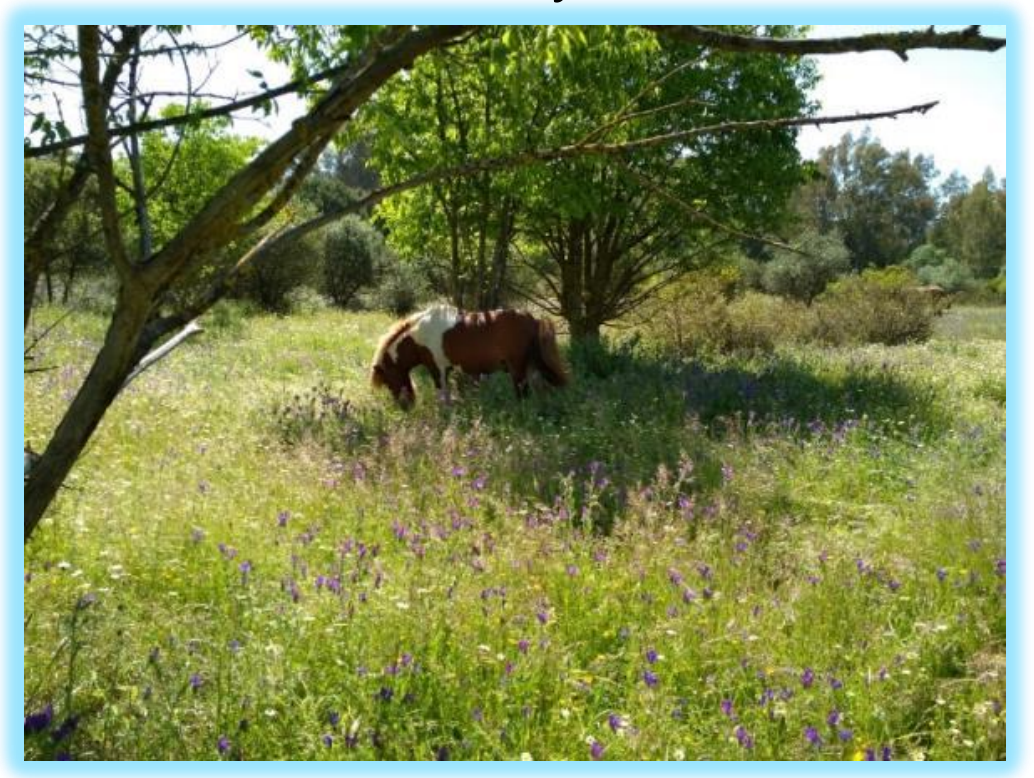

Sub-humid Mediterranean climate:

- Average temperatures: $9 \stackrel{\circ}{\circ} \mathrm{C}$ in winter and $29 \circ \mathrm{C}$ in summer.

- Irregular hydrological regime (summer droughts and flash floods during rainy periods).

Last year total precipitation: $403 \mathrm{~L} \mathrm{~m}^{-2}$
HPS - Highly Polluted Soil

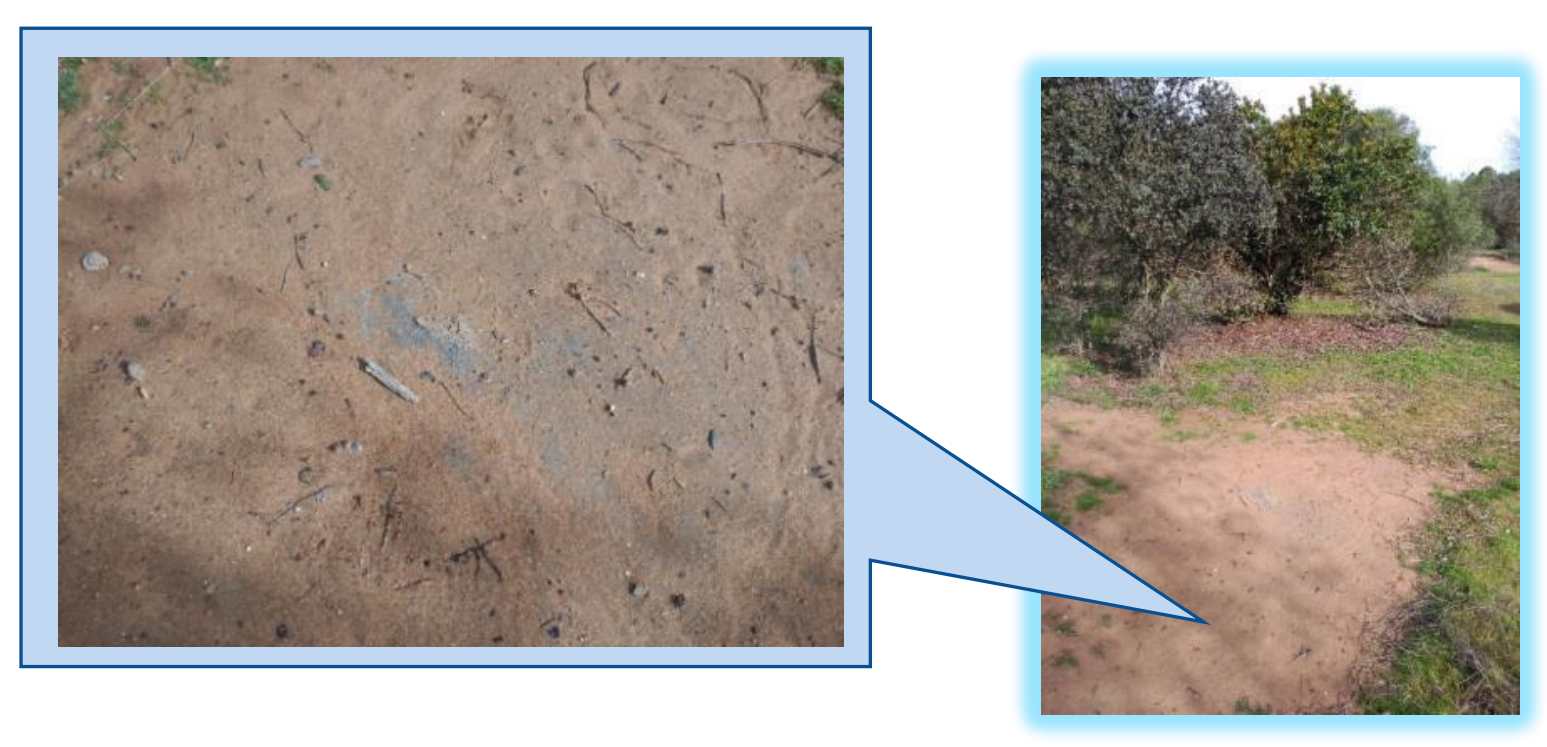




\section{Amendments: biochar}

pcampos@irnas.csic.es

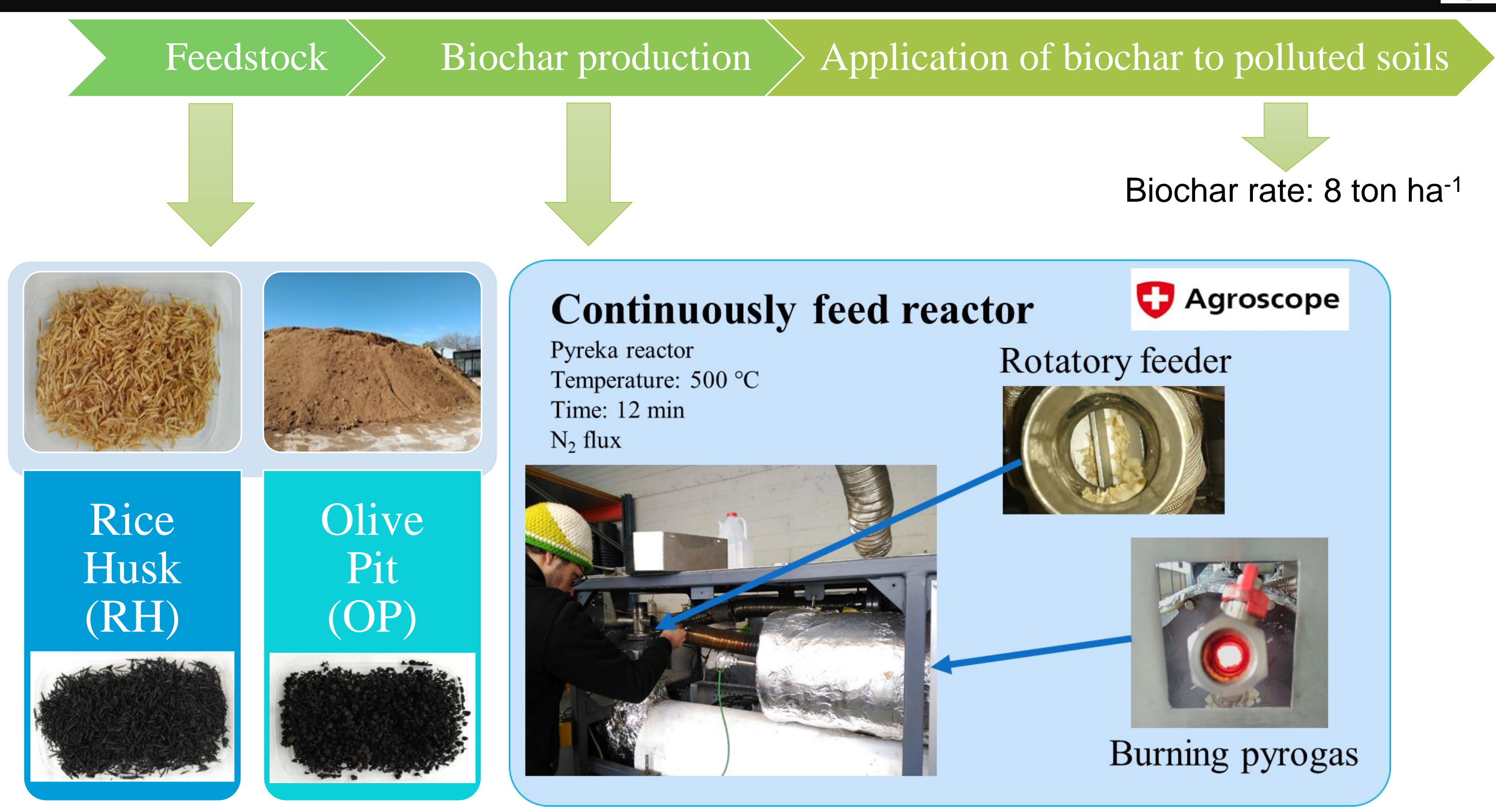




\begin{tabular}{|ccccc}
\hline & \multicolumn{4}{c}{$\mathrm{pH}$} \\
\cline { 2 - 5 } & \multicolumn{1}{c}{ Sample } & $\mathrm{t}_{6}$ & $\mathrm{t}_{12}$ & $\mathrm{t}_{20}$ \\
\cline { 2 - 5 } & C_HPS & $3.57 \pm 0.13 \mathrm{a}$ & $3.59 \pm 0.08 \mathrm{a}$ & $3.52 \pm 0.20 \mathrm{a}$ \\
& RHB_HPS & $3.64 \pm 0.18 \mathrm{a}$ & $3.68 \pm 0.21 \mathrm{a}$ & $3.77 \pm 0.17 \mathrm{a}$ \\
& OPB_HPS & $3.63 \pm 0.03 \mathrm{a}$ & $3.64 \pm 0.04 \mathrm{a}$ & $3.69 \pm 0.17 \mathrm{a}$ \\
\hline & C_MPS & $4.18 \pm 0.21 \mathrm{a}$ & $4.8 \pm 0.10 \mathrm{~b}$ & $4.87 \pm 0.20 \mathrm{~b}$ \\
& RHB_MPS & $4.75 \pm 0.01 \mathrm{~b}$ & $5.02 \pm 0.22 \mathrm{~b}$ & $5.07 \pm 0.18 \mathrm{~b}$ \\
& OPB_MPS & $4.74 \pm 0.54 \mathrm{~b}$ & $4.86 \pm 0.50 \mathrm{~b}$ & $5.11 \pm 0.20 \mathrm{~b}$ \\
\hline
\end{tabular}

a $\mathrm{pH}$ and water holding capacity.

- Increases of soil $\mathrm{pH}$ at $\leq 10 \mathrm{~cm}$ for MPS at a very short term.

- No significant variations after one year.

- Soil respiration

- MPS >> HPS.

Soil respiration $\left(\mu \mathrm{mol} \mathrm{CO}_{2} \mathrm{~m}^{-2} \mathrm{~s}^{-1}\right)$

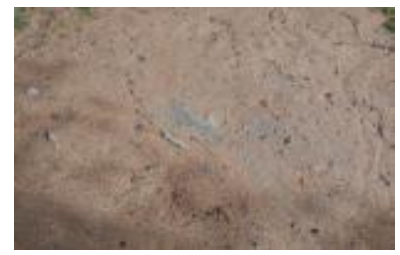

\begin{tabular}{|ccccc} 
& \multicolumn{4}{c}{ Soil respiration $\left(\mu \mathrm{mol} \mathrm{CO}_{2} \mathrm{~m}^{-2} \mathrm{~s}^{-1}\right)$} \\
\cline { 2 - 5 } & Sample & $\mathrm{t}_{6}$ & $\mathrm{t}_{12}$ & $\mathrm{t}_{20}$ \\
\cline { 2 - 5 } & C_HPS & $1.0 \pm 0.2 \mathrm{a}$ & $1.0 \pm 0.2 \mathrm{a}$ & $0.9 \pm 0.2 \mathrm{a}$ \\
& RHB_HPS & $1.0 \pm 0.3 \mathrm{a}$ & $0.8 \pm 0.2 \mathrm{a}$ & $0.6 \pm 0.3 \mathrm{a}$ \\
& OPB_HPS & $1.2 \pm 0.4 \mathrm{a}$ & $0.9 \pm 0.2 \mathrm{a}$ & $1.2 \pm 0.6 \mathrm{a}$ \\
\hline & C_MPS & $2.9 \pm 0.2 \mathrm{~b}$ & $1.2 \pm 0.8 \mathrm{a}$ & $3.9 \pm 0.2 \mathrm{~b}$ \\
\hline & RHB_MPS & $2.7 \pm 0.3 \mathrm{~b}$ & $1.3 \pm 0.7 \mathrm{a}$ & $3.3 \pm 0.4 \mathrm{~b}$ \\
& OPB_MPS & $3.1 \pm 0.3 \mathrm{~b}$ & $0.5 \pm 0.1 \mathrm{a}$ & $3.7 \pm 1.0 \mathrm{~b}$ \\
\hline
\end{tabular}

- The difference is reduced with time (effects of climatic conditions on MPS).

- No significative differences between control and biochar amended soils.

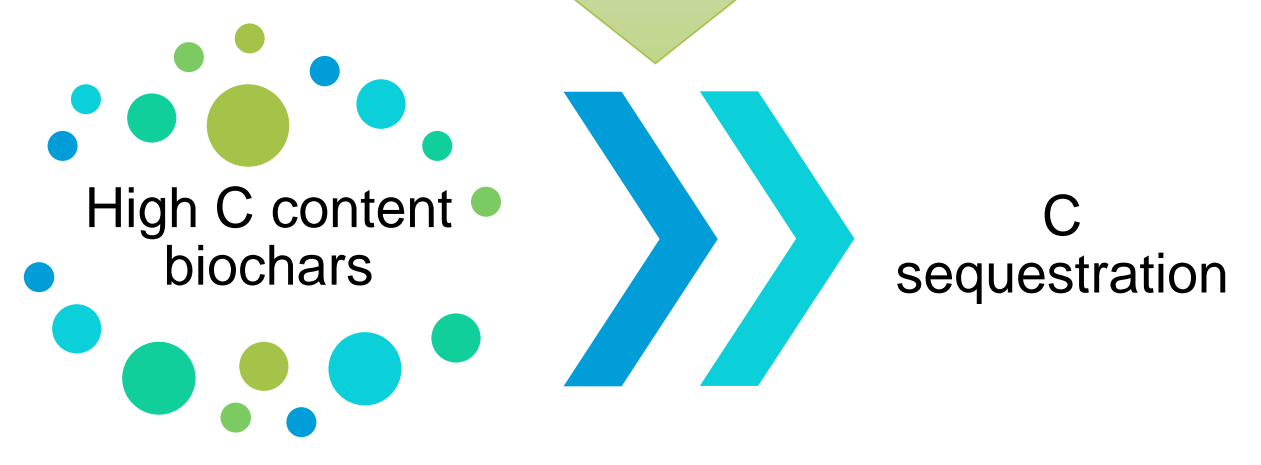




\section{2 months}

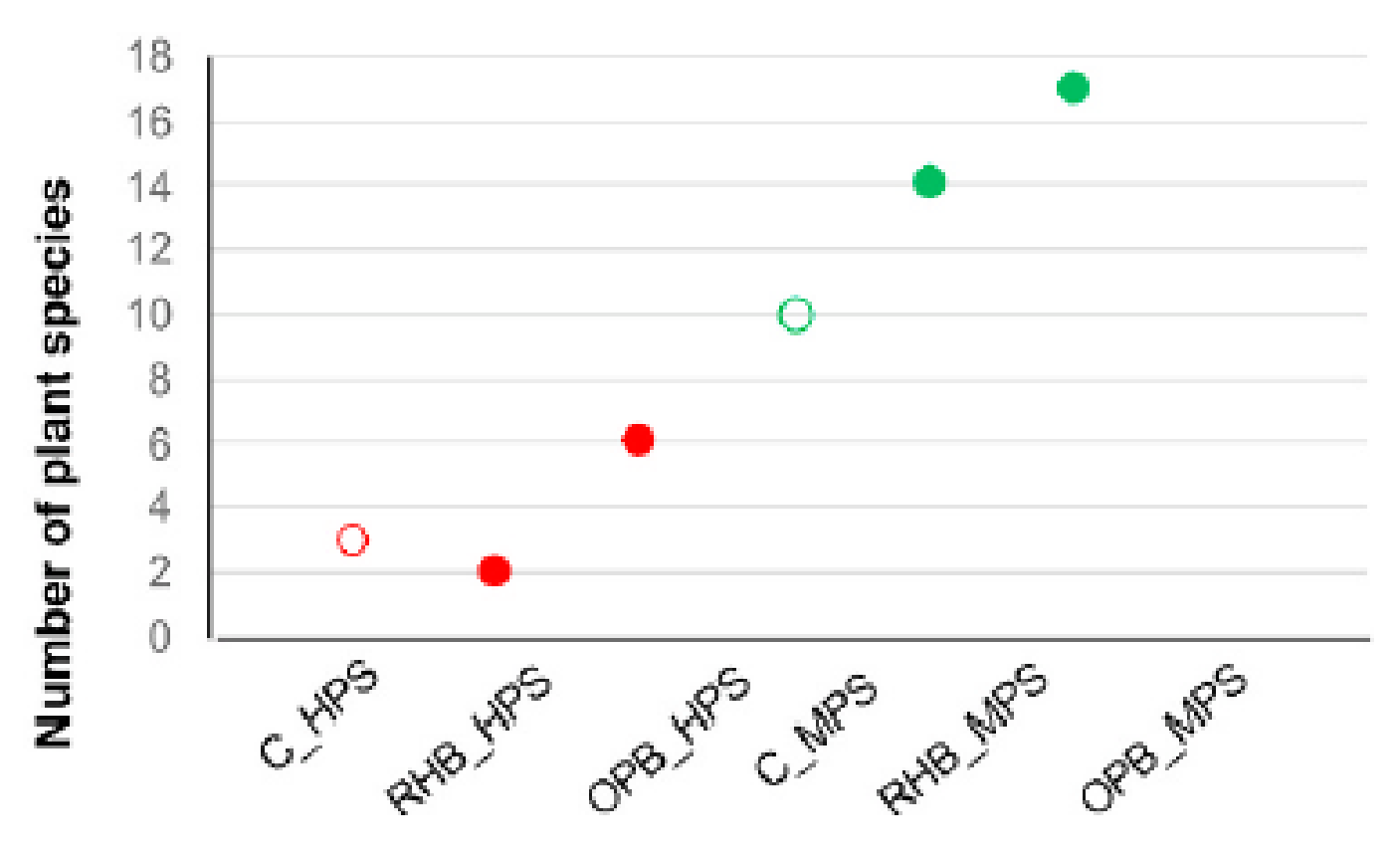

MPS showed greater number of plant species tan HPS.

Biochar application enhanced vegetation cover

Plant development (biomass production) and diversity was enhanced by biochar amendment.
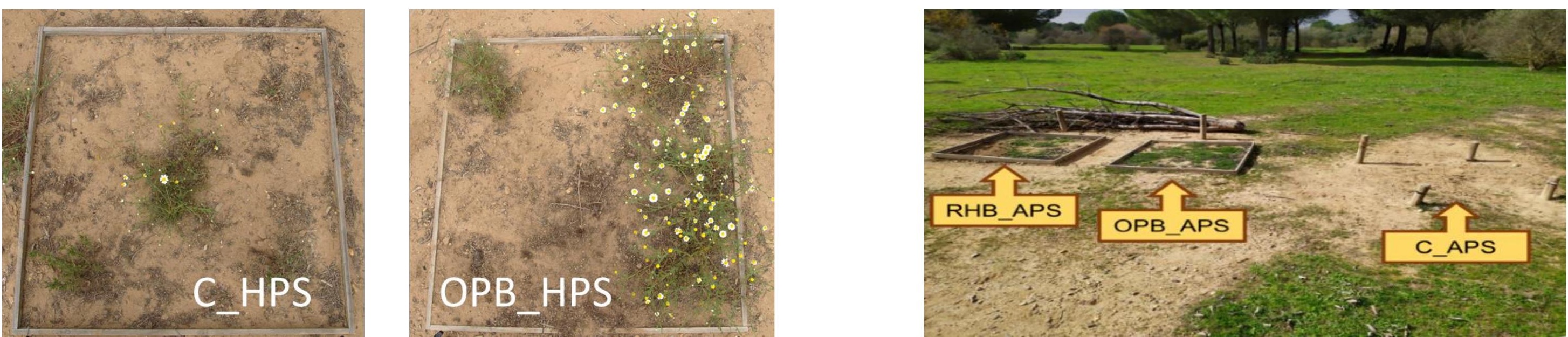

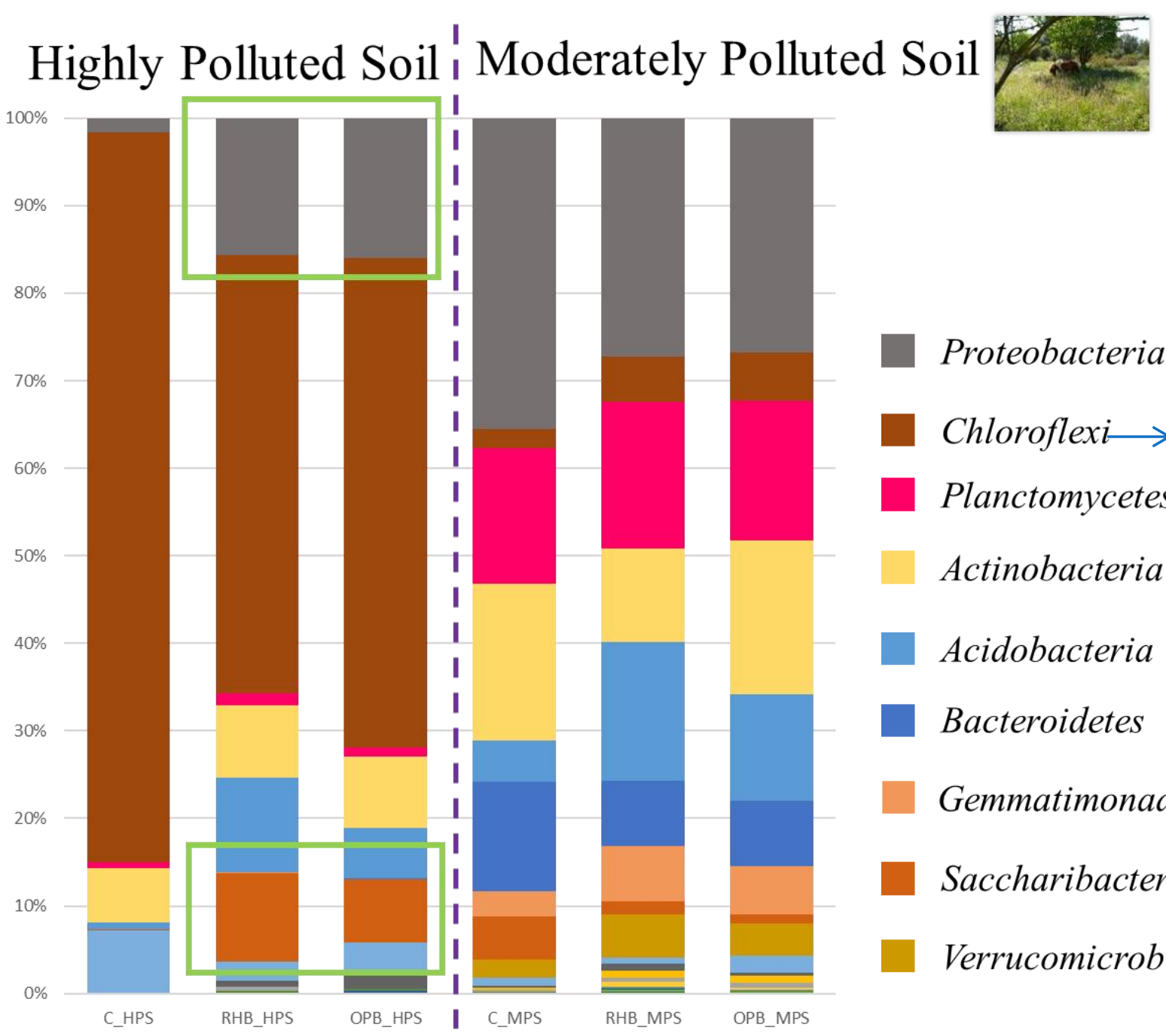

Proteobacteria

Chloroflexi

Planctomycetes

Ktedonobacteria (acid/extreme env.)

Actinobacteria

Acidobacteria

Bacteroidetes

Gemmatimonadetes

Saccharibacteria

Verrucomicrobia

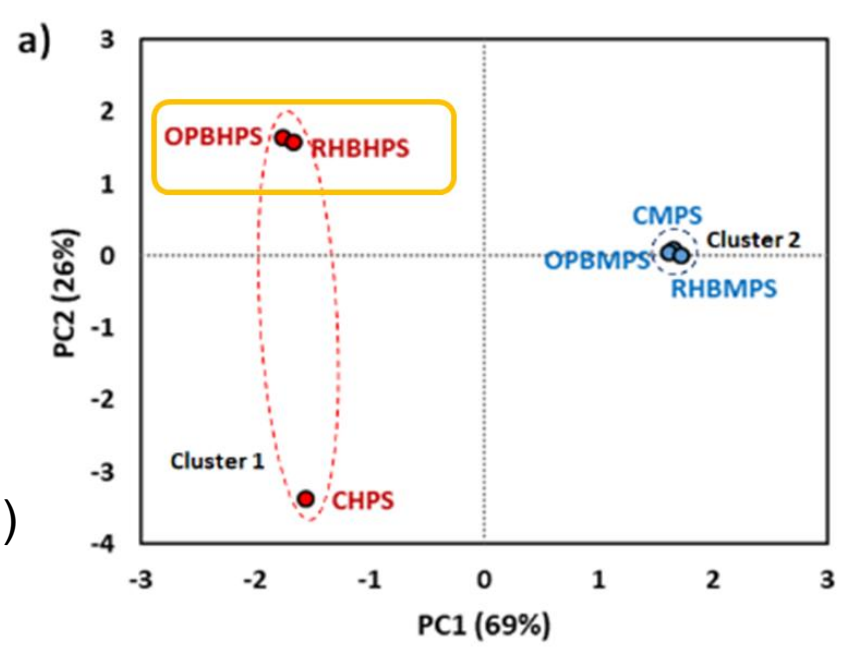

(6 meses)

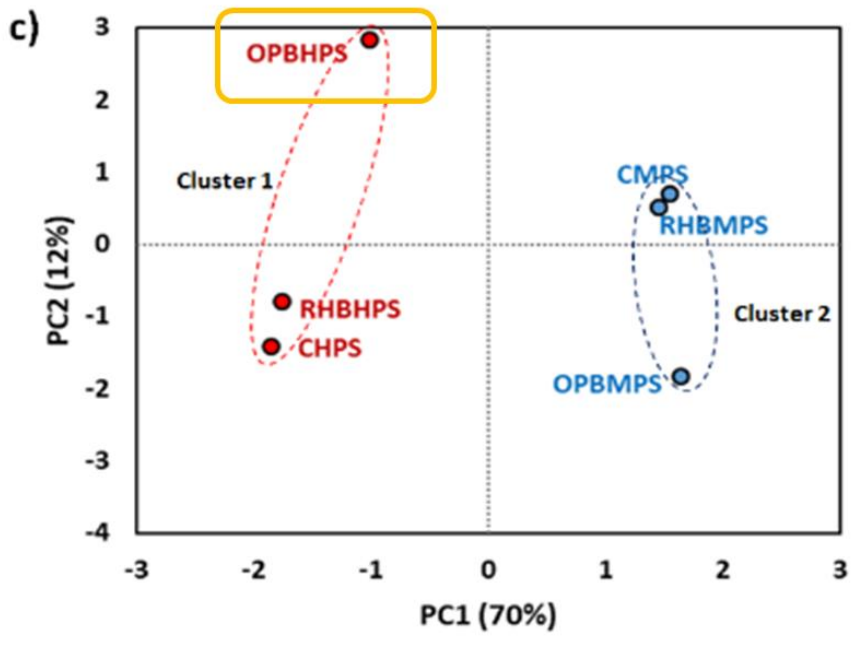

- Biochar application increased bacterial diversity decreasing extremophiles 


\section{Conclusions}

Some differences in soil properties, microbial population and plant diversity between MPS and HPS.

Differences were reduced with time and probably related with climate-seasonal effects.

After one year, 8 ton/ha of biochar was not enough to significatively change soil properties.

Biochar aplication modified the relative abundance of bacterial population in soils.

Further long-term analysis are required to investigate the effects of biochar on trace element polluted soil as the biochar continues to age (the experiment will continue for 2 years). 


\section{Thank you for your attention}

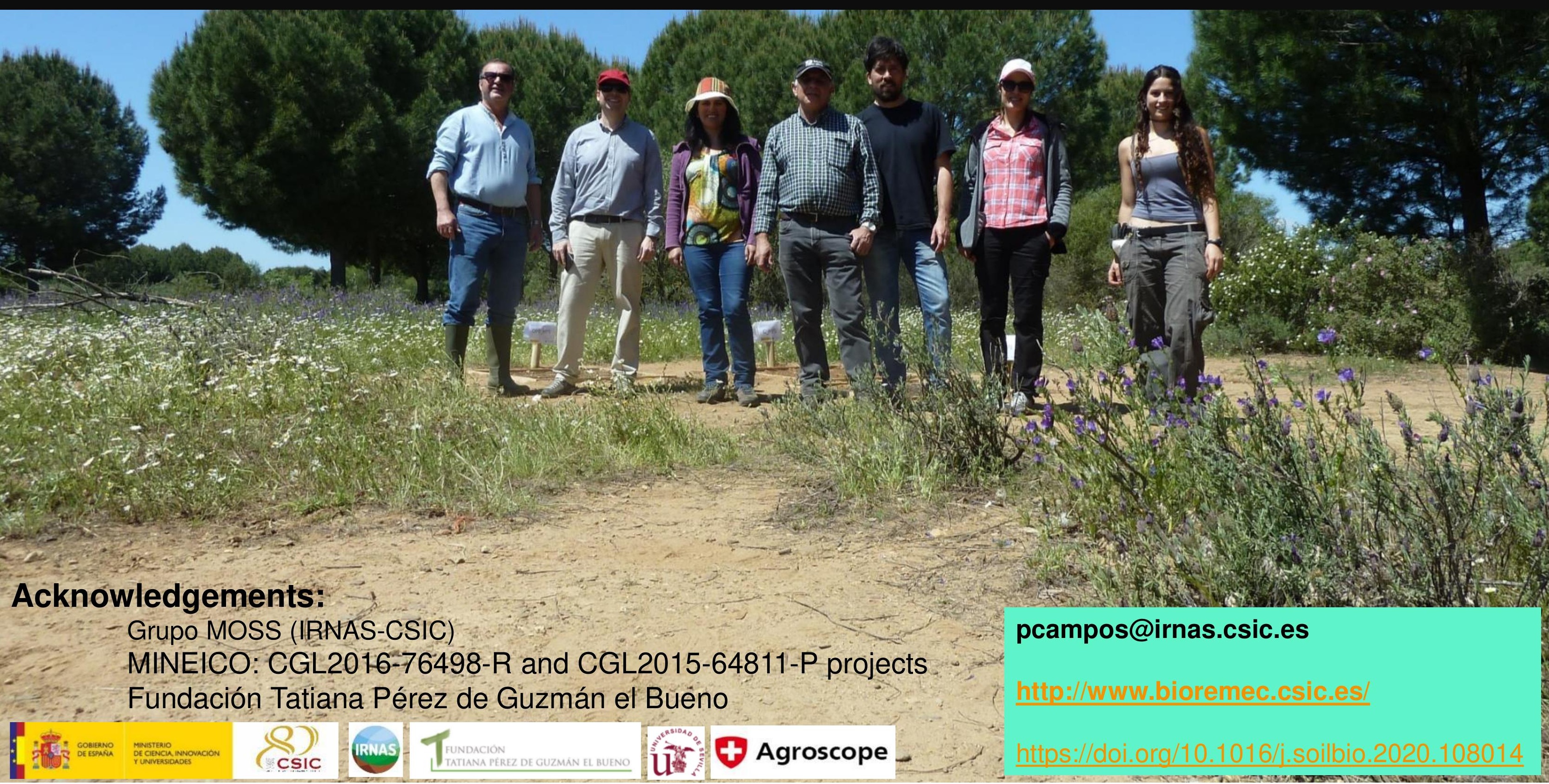

